\title{
2018 : Pas de meilleur moment pour être un interniste général au Canada
}

Au début des années 1990, lorsque je terminais ma formation à travers le programme de résidence, la médecine interne générale au Canada faisait face à une crise d'existence. D’un côté, les sousspécialités médicales prenaient le contrôle comme voie préférée pour les consultations des médecins de soins primaires. Un exemple type était qu'un patient recevait des soins d'un cardiologue pour son insuffisance cardiaque, un endocrinologue pour son diabète et un néphrologue pour son insuffisance rénale. De l'autre côté, la médecine interne générale était comprimée par lrascension de la médecine familiale comme spécialité à part entière, délimitant davantage le domaine généraliste. En tant que PGY-4 en médecine interne générale à l'Université de Toronto, je pouvais compter, d’une part, sur le nombre de professeurs qui se qualifiaient d'internistes généralistes, et d'autre part, il y avait encore moins de stagiaires dans ce domaine. Je me souviens distinctement d'un membre du corps professoral qui se plaignait d'avoir reçu une seule consultation externe d'un médecin de famille - toute l’année ! Au début de ma carrière, lorsque je travaillais comme interniste interne couvrant les services d'urgence des grands hôpitaux communautaires, les patients admis à l'hôpital étaient renvoyés le lendemain matin au sous-spécialiste le plus proche du diagnostic d'admission et, si nécessaire, d'autres sous-spécialistes ont été appelés pour aider avec des problèmes médicaux secondaires. Compte tenu de ces considérations, il n'est pas surprenant que la médecine interne générale soit l'une des destinations les moins souhaitables pour les résidents qui terminent leur formation de base en médecine interne. En effet, les options pour une carrière en médecine interne générale étaient plutôt limitées et incluaient la pratique dans une région géographique éloignée, manquaient de sous-spécialistes ou poursuivaient une carrière universitaire en milieu hospitalier où le champ de pratique réservé aux internistes généralistes était plutôt étroit (p. ex. dotation d'une équipe de consultation péri-opératoire).

Si le début des années 1990 peut être considéré comme le point le plus bas de la médecine interne générale au Canada, je suis heureux de dire que les années 2010 sont une période où le présent est brillant et le futur est encore plus prometteur. Qu'est-ce qui a conduit à cette transformation genre phénix de la médecine interne générale ? Premièrement, le Collège royal des médecins et chirurgiens du Canada a officiellement reconnu en 2010 que la médecine interne générale était une sous-spécialité bona fide et que les exigences en matière de formation seraient ajustées en conséquence. En effet, les internistes généralistes possèdent létendue des connaissances et de l'expertise qui manqueraient à d'autres sous-spécialités et, contrairement à nos collègues de soins primaires, ont une expertise approfondie pour de nombreuses affections courantes comme l'insuffisance cardiaque, la broncho-pneumopathie chronique obstructive, le diabète, l'insuffisance rénale et hépatique pour n’en nommer que quelques-uns. Deuxièmement, le Collège royal a créé des programmes de diplômes appelés domaines de compétence ciblée (ou «DCC»), qui sont devenus disponibles, dont un grand nombre pouvaient être intégrés à la voie de la médecine interne générale. Une des plus récentes DCC, qui me tient particulièrement à cœur, est la médecine de la thrombose chez l'adulte. Cela commencera à recruter des stagiaires cette année et, compte tenu de sa participation à de multiples organismes, s'intégrera bien avec une pratique en médecine interne générale. D’autres DCC disponibles incluent l’hépatologie des adultes, la médecine transfusionnelle et la transplantation d’organes solides chez les adultes. En 2018, les internistes généralistes sont bien placés pour concevoir leur pratique afin d'inclure un domaine d'expertise plus spécialisé, que ce soit par le biais d'un programme officiel de DCC ou en dehors de celui-ci. Enfin, et peut-être le plus important, c'est la reconnaissance tardive de la valeur accordée aux internistes généralistes par les hôpitaux, les établissements de soins primaires et les établissements d’enseignement.

Il est donc agréable, surtout pour beaucoup d'entre nous d'un millésime plus âgé, de voir la médecine interne générale comme une destination de choix. Il est également agréable de voir en 2018 ce que beaucoup d'entre nous ont connu depuis des décennies: la médecine interne générale offre un parcours de carrière unique qui combine des éléments de sous-spécialité avec le génie généraliste que nous apprécions tant. Il n'y a vraiment pas de meilleur moment pour devenir interniste général au Canada.

DOI: 10.22374/cjgim.v13i3.316
James Douketis MD Editeur em chef

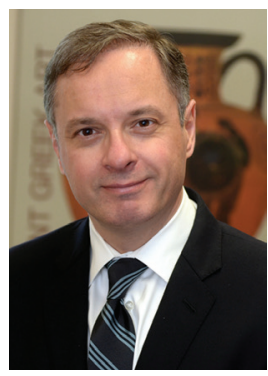

